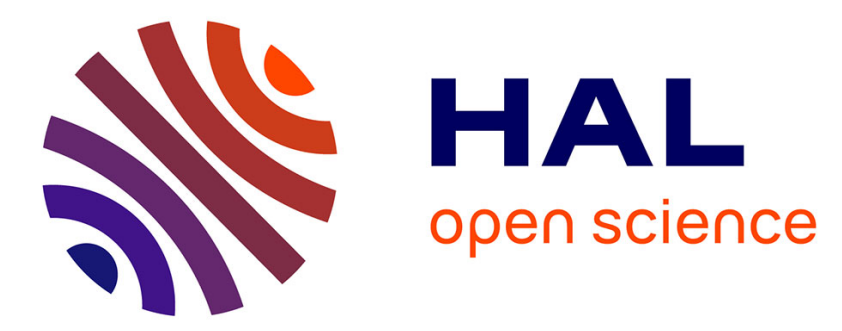

\title{
Shrinking complexity of scheduling dependencies in LS-SVM based LPV system identification
}

\author{
René Duijkers, Roland Tóth, Dario Piga, Vincent Laurain
}

\section{To cite this version:}

René Duijkers, Roland Tóth, Dario Piga, Vincent Laurain. Shrinking complexity of scheduling dependencies in LS-SVM based LPV system identification. 53rd IEEE Conference on Decision and Control, CDC 2014, Dec 2014, Los Angeles, United States. hal-01088014

\section{HAL Id: hal-01088014 https://hal.science/hal-01088014}

Submitted on 4 Dec 2014

HAL is a multi-disciplinary open access archive for the deposit and dissemination of scientific research documents, whether they are published or not. The documents may come from teaching and research institutions in France or abroad, or from public or private research centers.
L'archive ouverte pluridisciplinaire $\mathbf{H A L}$, est destinée au dépôt et à la diffusion de documents scientifiques de niveau recherche, publiés ou non, émanant des établissements d'enseignement et de recherche français ou étrangers, des laboratoires publics ou privés. 


\title{
Shrinking Complexity of Scheduling Dependencies in LS-SVM Based LPV System Identification
}

\author{
René Duijkers, Roland Tóth, Dario Piga and Vincent Laurain
}

\begin{abstract}
In the past years, Linear Parameter-Varying (LPV) identification has rapidly evolved from parametric identification methods to nonparametric methods allowing the relaxation of restrictive assumptions. For example, Least-Square Support Vector Machines (LS-SVMs) offer an attractive way of estimating LPV models directly from data without requiring from the user to specify the functional dependencies of the model coefficients on the scheduling variable. These methods have also been recently extended in order to automatically determine the model order directly from data by the help of regularization. Nonetheless, despite all these recent improvements, LPV identification methods still require some strong $a$ priori such as $i$ ) the dependencies are static or dynamic, $i i)$ it is known which variables are considered to be the scheduling or iii) all coefficient functions of the underlaying system depend on all scheduling variables. This prevents the complexity of the scheduling dependency of the model to be shrunk gradually and independently until an optimal bias-variance trade off is found. In this paper, a novel reformulation of the LPV LS-SVM approach is proposed which, besides of the non-parametric estimation of the coefficient functions, achieves data-driven coefficient complexity selection via convex optimization. The properties of the introduced approach are illustrated by a simulation study.
\end{abstract}

\section{INTRODUCTION}

The need to efficiently develop high-performance control solutions for industrial applications with position dependency, varying operating conditions or nonlinear/timevarying system behavior, has resulted in an increasing interest for model structures that can represent the behavior of such systems with a linear representation, allowing the use of powerful control synthesis tools. Linear parameter-varying (LPV) models, introduced in [1], have become a promising way of answering to this need in control engineering [2].

An efficient way to estimate LPV models in general is using a least-square support vector machine (LS-SVM) approach [3]. This method allows to model nonlinear systems with an LPV input-output (IO) model without requiring from the user to specify the functional dependencies of the model coefficients on the scheduling variables. This is achieved by

R. Duijkers and R. Tóth are with the Department of Electrical Engineering, Eindhoven University of Technology, P.O. Box 5135600 MB Eindhoven, The Netherlands. E-mail: $r$.duijkersestudent.tue.nl; r.toth@tue.nl.

Dario Piga is with the Dalle Molle Institute for Artificial Intelligence Research, Scuola Universitaria Professionale della Svizzera Italiana, Galleria 1, 6928 Manno, Switzerland. E-mail: dario.piga@supsi.ch

V. Laurain is with the Centre de Recherche en Automatique de Nancy, Université de Lorraine, CNRS, 2 rue Jean Lamour, 54519 Vandoeuvre-lès-Nancy Cedex, France. E-mail: vincent. lauraineuniv-lorraine.fr.

This work was supported by the Netherlands Organization for Scientific Research (NWO, grant. no.: 639.021.127). using the dual solution of a regularized $\ell_{2}$ loss function. The resulting approach provides a direct nonparametric estimation of the dependencies from data under general noise conditions, where the estimate is obtained by solving a set of linear equations [4], [5].

A particular handicap of the of LS-SVM based LPV identification approaches is that the non-parametric estimation is governed by the so-called Kernel functions, which are assumed to depend on all scheduling variables to avoid prior structural specification of the dependencies. However, this corresponds to a prejudice that all coefficient functions of the underlaying system depend on all of these variables which is not the case in most practical applications. Commonly, it is not known beforehand which variables play a significant role in the dependence and which variables can be excluded. Taking all of them into account leads to an over-parametrization problem which results in an increased variance and complexity of the estimated models.

In this paper, the aim is to tackle this overparameterization by estimating the underlying structure of the process. To achieve this, we propose a regularization based LS-SVM method which penalizes coefficient functions that are insensitive w.r.t. variations of the scheduling variable. The introduced penalty on the coefficient functions gradually shrinks the non-dependent functions to constants (including zero), thereby revealing the underlying structure and reducing complexity in terms of model order and dependency. In contrast with a previous work [6], the proposed method does not directly penalize the amplitude of the coefficient functions, but it shrinks the sensitivity (i.e., the derivative) of the functions with respect to the scheduling signals to zero. This means that the dependency can decrease in terms of complexity rather than just completely vanishing as in the sparse estimator approach proposed in [6]. This is highly beneficial when using the estimated models for control synthesis, commonly requiring low coefficient complexity.

The paper is organized as follows: first, in Section II-A, the LPV context in which the estimation problem is addressed is presented. Then, Sections II-B to II-D describe the identification problem and the solution using the LSSVM estimator and the proposed regularization structure. Finally, the performance of the approach is demonstrated in a Monte-Carlo study in Section III and conclusions are drawn in Section IV.

\section{LPV MODEL SELECTION}

In this section, the proposed approach to determine the structure and the underlaying dependencies of an LPV-IO 
model is detailed. Once the LPV model to be identified and its variables are introduced, the proposed regularized identification problem is presented: the objective of coefficient dependency shrinkage is expressed by introducing an additional term into the regularized $\ell_{2}$ prediction-error cost function. Finally an LS-SVM estimator is formulated and it is shown that the introduced regularization term allows a gradual shrinking of the complexity of the estimated functional dependencies.

\section{A. Model structure}

For the sake of brevity, the simplest form of LPV models used in the discrete-time IO identification framework is considered, which is the autoregressive with exogenous input (ARX) structure, defined, in the single-input single-output (SISO) case, as

$y(k)=\sum_{i=1}^{n_{\mathrm{a}}} a_{i}(p(k)) y(k-i)+\sum_{j=0}^{n_{\mathrm{b}}} b_{j}(p(k)) u(k-j)+e(k)$.

Here, $k \in \mathbb{Z}$ denotes the discrete time, $e(k) \in \mathbb{R}$ is assumed to be a zero-mean white noise, $u(k) \in \mathbb{R}$ and $y(k) \in \mathbb{R}$ represent the measured input and output signals of the system to be identified, respectively, and $p(k) \in \mathbb{R}^{n_{\mathrm{p}}}$ is the socalled scheduling variable, which ranges in a compact set $\mathbb{P} \subseteq \mathbb{R}^{n_{\mathrm{p}}}$ and, according to the literature (see, e.g., [2]), it is assumed to be measurable. According to the LPV nature of the model, the coefficients $a_{i}$ and $b_{j}$ are seen as functions of the scheduling signal $p$ and they are intended to describe the varying linear dynamical relation between $u$ and $y$. For clarity of exposition, in this paper, the model coefficients $a_{i}$ and $b_{j}$ are considered to be differentiable and to have only static dependence on $p$, i.e., $a_{i}$ and $b_{j}$ only depend on the value of $p$ at time $k$. Note that in parametric identification of LPV systems, the dependence of the coefficient functions $a_{i}$ and $b_{j}$ on the scheduling parameter $p$ is commonly parameterized in terms of a linear combination of a finite number of a priori chosen basis functions (linear basis, monomials, etc.) in the variable $p(k)$, see e.g., [7], [8]. On the other hand, in this contribution, the dependence of $a_{i}$ and $b_{j}$ on the scheduling variable $p$ is not $a$ priori parameterized.

Rewrite the model structure (1) as

$$
y(k)=\sum_{i=1}^{n_{\mathrm{g}}} a_{i}(p(k)) x_{i}(k)+e(k),
$$

where $x_{i}(k)$ denotes the $i$-th component of the $n_{\mathrm{g}}=n_{\mathrm{a}}+$ $n_{\mathrm{b}}+1$-dimensional vector $x(k)$ defined as

$$
x(k)=\left[\begin{array}{lllll}
y(k-1) & \ldots & y\left(k-n_{\mathrm{a}}\right) u(k) & \ldots & u\left(k-n_{\mathrm{b}}\right)
\end{array}\right]^{\top}
$$

and, with an abuse of notation, $\left\{a_{i}(\cdot)\right\}_{i=1}^{n_{\mathrm{g}}}$ stands for all the associated coefficient functions, according to (1). Similar to the parametric case, the coefficient functions $a_{i}$ are considered to have a hypothetical parameterization

$$
a_{i}(\cdot)=\omega_{i}^{\top} \phi_{i}(\cdot)
$$

where each $\phi_{i}$ is a nonlinear map from the scheduling parameter space $\mathbb{P}$ to a high-dimensional space, the socalled feature space $\mathbb{R}^{n_{\mathrm{H}}} \cdot \omega_{i}$ is an $n_{\mathrm{H}}$-dimensional vector of constant parameters. In contrast with the parametric case, the structural dependence of the coefficients $\phi_{i}$ on $p$ is assumed to be a priori unknown and the dimension $n_{\mathrm{H}}$ of the parameter vector $\omega_{i}$ is potentially infinite. One of the originality of this work with respect to usual assumptions is to consider that $a_{i}($.$) might depend only on a dimensional$ subset of $p(k)$. Should the elements of $p(k)$ correspond to delayed version of a one dimensional scheduling variable, the presented approach can be seen as a way to estimate parsimoniously dynamical or delayed dependencies. Should the elements of $p(k)$ be different scheduling variables, the aim would be to determine which dependencies best suit for each nonlinear dependency or whether the coefficients are scheduling dependent at all.

\section{B. Identification problem}

The goal is to estimate the dependence of the coefficient functions $a_{i}(\cdot)$ on the scheduling variable $p$ accurately, but, at the same time, minimize the "complexity" of these estimated functions till an appropriate trade off between the prediction error (fit w.r.t. the data) and variation of the functions over $\mathbb{P}$ is found. The latter corresponds to a particular bias/variance trade off. This is done by solving an optimization problem using a set of $N$ data points $\mathcal{D}_{N}=\{(u(k), p(k), y(k))\}_{k=1}^{N}$, generated by the system to be identified. This system is assumed to lay inside of the model set corresponding to (1).

Like in non-regularized LS-SVM identification approaches, e.g., [3], [9], [10], the optimization problem uses a cost function penalizing the 2-norm of the parameter vectors $\omega_{i}$ and the residual $e(k)=y(k)-\sum_{i=1}^{n_{\mathrm{g}}} \omega_{i}^{\top} \phi_{i}(p(k)) x_{i}(k)$ corresponding to the one-step-ahead prediction error by (1). Because we aim at estimating the dependence of $\phi_{i}$ on the scheduling variable $p$ with a minimal complexity, which translates to the expectation of a smooth variation of each $a_{i}$ over $\mathbb{P}$, an additional term to penalize the norm of the sensitivity of each $\phi_{i}$ towards each dimension of $p$ is introduced. This results in the following identification criterion, i.e., cost function:

$$
\mathcal{W}(\omega, e)=\frac{1}{2}\|\omega\|_{\ell_{2}}^{2}+\frac{\gamma_{1}}{2}\|e(k)\|_{\ell_{2}}^{2}+\gamma_{2} \sum_{i=1}^{n_{\mathrm{g}}} \sum_{j=1}^{n_{\mathrm{p}}}\left\|\frac{\partial \omega_{i}^{\top} \phi_{i}(p)}{\partial p_{j}}\right\|_{\ell_{\infty}}
$$

where $\gamma_{1}>0$ and $\gamma_{2}>0$ represent the tuning hyperparameters specified by the user, commonly required in regularized optimization.

Due to the differentiability assumption, the norm penalizing the sensitivity can be written as

$$
\left\|\frac{\partial \omega_{i}^{\top} \phi_{i}(p)}{\partial p_{j}}\right\|_{\ell_{\infty}}=\sup _{p \in \mathbb{P}}\left|\frac{\partial \omega_{i}^{\top} \phi_{i}(p)}{\partial p_{j}}\right| .
$$

Let us define the index set $\mathbb{I}_{s}^{\tau}=\{z \in \mathbb{Z} \mid s \leq z \leq \tau\}$ and the set $\mathcal{P}=\left\{\begin{array}{lll}\bar{p}_{1}^{\left(s_{1}\right)} \ldots & \bar{p}_{n_{\mathrm{p}}}^{\left(s_{n_{\mathrm{p}}}\right)}\end{array}\right] \in \mathbb{P} \mid$ with $s_{1} \in$ $\left.\mathbb{I}_{1}^{n_{\mathrm{s}}}, \ldots, s_{n_{\mathrm{p}}} \in \mathbb{I}_{1}^{n_{\mathrm{s}}}\right\}$ which corresponds to an (equidistant) gridding of $\mathbb{P}$ with $n_{\mathrm{s}}$ points along each dimension. Using these grid points, we can calculate a lower-bound approxi- 
mation of the sensitivity norm:

$$
\sup _{p \in \mathbb{P}}\left|\frac{\partial \omega_{i}^{\top} \phi_{i}(p)}{\partial p_{j}}\right| \geq \max _{\bar{p} \in \mathcal{P}}\left|\frac{\partial \omega_{i}^{\top} \phi_{i}(p)}{\partial p_{j}}\right|_{p=\bar{p}} .
$$

By introducing the slack variables $\left\{r_{i, j}\right\}_{i=1, j=1}^{n_{\mathrm{g}}, n_{\mathrm{p}}}$, we can formulate the identification problem in terms of a quadratic program:

$$
\begin{gathered}
\min _{w, r, e} \frac{1}{2} \sum_{i=1}^{n_{\mathrm{g}}} \omega_{i}^{\top} \omega_{i}+\frac{\gamma_{1}}{2} \sum_{k=1}^{N} e^{2}(k)+\gamma_{2} \sum_{i=1}^{n_{\mathrm{g}}} \sum_{j=1}^{n_{\mathrm{p}}} r_{i, j} \\
\text { s.t. } \quad e(k)=y(k)-\sum_{i=1}^{n_{\mathrm{g}}} \omega_{i}^{\top} \phi_{i}(p(k)) x_{i}(k), \quad k \in \mathbb{I}_{1}^{N} \\
-r_{i, j} \leq\left.\frac{\partial \omega_{i}^{\top} \phi_{i}(p)}{\partial p_{j}}\right|_{p=\bar{p}} \leq r_{i, j} \\
i \in \mathbb{I}_{1}^{n_{\mathrm{g}}}, j \in \mathbb{I}_{1}^{n_{\mathrm{p}}}, \bar{p} \in \mathcal{P}
\end{gathered}
$$

Note that the parameters $\omega_{i}$ in (7) cannot be computed since it would require an explicit representation of the feature maps $\phi_{i}$.

\section{Dual formulation}

Since we cannot estimate both the parameters and the feature maps together, the dual formulation of (7) is considered. For this purpose, let us define the Lagrangian as

$$
\begin{aligned}
& \mathcal{L}\left(\omega, e, r, \alpha, \beta^{+}, \beta^{-}\right)= \\
& \frac{1}{2} \sum_{i=1}^{n_{\mathrm{g}}} \omega_{i}^{\top} \omega_{i}+\frac{\gamma_{1}}{2} \sum_{k=1}^{N} e^{2}(k)+\gamma_{2} \sum_{i=1}^{n_{\mathrm{g}}} \sum_{j=1}^{n_{\mathrm{p}}} r_{i, j} \\
& -\sum_{k=1}^{N} \alpha_{k}\left(e(k)-y(k)+\sum_{i=1}^{n_{\mathrm{g}}} \omega_{i}^{\top} \phi_{i}(p(k)) x_{i}(k)\right) \\
& -\sum_{i=1}^{n_{\mathrm{g}}} \sum_{j=1}^{n_{\mathrm{p}}} \sum_{m=1}^{M} \beta_{i, j, m}^{+}\left(r_{i, j}-\frac{\partial \omega_{i}^{\top} \phi_{i}\left(\bar{p}_{m}\right)}{\partial p_{j}}\right) \\
& -\sum_{i=1}^{n_{\mathrm{g}}} \sum_{j=1}^{n_{\mathrm{p}}} \sum_{m=1}^{M} \beta_{i, j, m}^{-}\left(r_{i, j}+\frac{\partial \omega_{i}^{\top} \phi_{i}\left(\bar{p}_{m}\right)}{\partial p_{j}}\right) .
\end{aligned}
$$

Here $\alpha=\left\{\alpha_{k}\right\}_{k=1}^{N}, \beta^{+}=\left\{\beta_{i, j, m}^{+}\right\}_{i=1, j=1, m=1}^{n_{\mathrm{g}}, n_{\mathrm{p}}, M}$ and $\beta^{-}=$ $\left\{\beta_{i, j, m}^{-}\right\}_{i=1, j=1, m=1}^{n_{\mathrm{g}}, n_{\mathrm{p}}, M}$ are the Lagrangian multipliers and $\mathcal{P}=$ $\left\{\bar{p}_{m}\right\}_{m=1}^{M}$ is a set containing all $\left(M=n_{\mathrm{p}} \times n_{\mathrm{s}}\right)$ grid points. The term $\frac{\partial \omega_{i}^{\top} \phi_{i}\left(\bar{p}_{m}\right)}{\partial p_{j}}$ has been used as a short notation for $\left.\frac{\partial \omega_{i}^{\top} \phi_{i}(p)}{\partial p_{j}}\right|_{p=\bar{p}_{m}}$.

Next, the Lagrange dual function $g\left(\alpha, \beta^{+}, \beta^{-}\right)$is defined as the infimum of $\mathcal{L}\left(\omega, e, r, \alpha, \beta^{+}, \beta^{-}\right)$over $(\omega, e, r)$, i.e.,

$$
g\left(\alpha, \beta^{+}, \beta^{-}\right)=\inf _{\omega, e, r} \mathcal{L}\left(\omega, e, r, \alpha, \beta^{+}, \beta^{-}\right)
$$

Since $\mathcal{L}\left(\omega, e, r, \alpha, \beta^{+}, \beta^{-}\right)$is a convex quadratic function of $(\omega, e, r)$, the infimum of the Lagrange dual function $g\left(\alpha, \beta^{+}, \beta^{-}\right)$can be computed from the (necessary and sufficient) optimality conditions:

$$
\begin{aligned}
& \frac{\partial \mathcal{L}}{\partial \omega_{i}}=0 \rightarrow \omega_{i}=\sum_{k=1}^{N} \alpha_{k} \phi_{i}(p(k)) x_{i}(k) \\
&+\sum_{j=1}^{n_{\mathrm{p}}} \sum_{m=1}^{M} \frac{\partial \phi_{i}\left(\bar{p}_{m}\right)}{\partial p_{j}}\left(\beta_{i, j, m}^{-}-\beta_{i, j, m}^{+}\right) \\
& \frac{\partial \mathcal{L}}{\partial e}=0 \rightarrow \alpha_{k}=\gamma_{1} e(k) \\
& \frac{\partial \mathcal{L}}{\partial r_{i, j}}=0 \rightarrow \gamma_{2}=\sum_{m=1}^{M}\left(\beta_{i, j, m}^{-}+\beta_{i, j, m}^{+}\right)
\end{aligned}
$$

Here, condition (10a) needs to be satisfied for all $i \in \mathbb{I}_{1}^{n_{\mathrm{g}}}$, (10b) for all $k \in \mathbb{I}_{1}^{N}$ and condition (10c) for all $i \in \mathbb{I}_{1}^{n_{\mathrm{g}}}$ and $j \in \mathbb{I}_{1}^{n_{\mathrm{p}}}$. Substitution of the optimality conditions (10a)-(10c) into the Lagrangian (8) gives the Lagrange dual function as given in (11).

In (11), $X_{i}$ is a diagonal matrix whose diagonal entries are $x_{i}(1), \cdots, x_{i}(N) \cdot \Phi_{i}^{(N)}, \Delta_{i}^{\left(M \times n_{\mathrm{p}}\right)}, \beta^{+}, \beta^{-}$are defined as

$$
\begin{aligned}
& \Delta_{i}^{\left(M \times n_{\mathrm{p}}\right)}=\left[\begin{array}{llll}
\frac{\partial \phi_{i}\left(\bar{p}_{1}\right)}{\partial p_{1}} & \ldots & \frac{\partial \phi_{i}\left(\bar{p}_{1}\right)}{\partial p_{n_{\mathrm{p}}}} & \ldots
\end{array}\right. \\
& \left.\ldots \quad \frac{\partial \phi_{i}\left(\bar{p}_{M}\right)}{\partial p_{1}} \quad \ldots \quad \frac{\partial \phi_{i}\left(\bar{p}_{M}\right)}{\partial p_{n_{\mathrm{p}}}}\right]
\end{aligned}
$$

and

$$
\begin{aligned}
\Phi_{i}^{(N)} & =\left[\begin{array}{lllll}
\phi_{i}(1) & \phi_{i}(2) & \ldots & \phi_{i}(N)
\end{array}\right], \\
\beta^{+} & =\left[\begin{array}{llllll}
\beta_{i, 1,1}^{+} & \ldots & \beta_{i, n_{\mathrm{p}}, 1}^{+} & \beta_{i, 1,2}^{+} & \ldots & \beta_{i, n_{\mathrm{p}}, M}^{+}
\end{array}\right]^{\top}, \\
\beta^{-} & =\left[\begin{array}{llllll}
\beta_{i, 1,1}^{-} & \ldots & \beta_{i, n_{\mathrm{p}}, 1}^{-} & \beta_{i, 1,2}^{-} & \ldots & \beta_{i, n_{\mathrm{p}}, M}^{-}
\end{array}\right]^{\top} .
\end{aligned}
$$

The Lagrange dual problem associated with (7) is now given by the optimization problem:

$$
\begin{aligned}
& \min _{\alpha, \beta^{+}, \beta^{-}}-g\left(\alpha, \beta^{+}, \beta^{-}\right) \\
& \text {s.t. } \quad \gamma_{2}=\sum_{m=1}^{M}\left(\beta_{i, j, m}^{-}+\beta_{i, j, m}^{+}\right) \\
& \quad \beta_{i, j, s_{1}, m}^{+}, \beta_{i, j, m}^{-} \geq 0, \quad i \in \mathbb{I}_{1}^{n_{\mathrm{g}}}, j \in \mathbb{I}_{1}^{n_{\mathrm{p}}}, m \in \mathbb{I}_{1}^{M}
\end{aligned}
$$

Note that (13) is a convex optimization problem since the Lagrange dual function $g\left(\alpha, \beta^{+}, \beta^{-}\right)$is concave. In fact, the matrix

$$
\Omega_{i}=\left[\begin{array}{cc}
X_{i}\left(\Phi_{i}^{(N)}\right)^{\top} \Phi_{i}^{(N)} X_{i} & X_{i}\left(\Phi_{i}^{(N)}\right)^{\top} \Delta_{i}^{\left(M \times n_{\mathrm{p}}\right)} \\
\left(\Delta_{i}^{\left(M \times n_{\mathrm{p}}\right)}\right)^{\top} \Phi_{i}^{(N)} X_{i} & \left(\Delta_{i}^{\left(M \times n_{\mathrm{p}}\right)}\right)^{\top} \Delta_{i}^{\left(M \times n_{\mathrm{p}}\right)}
\end{array}\right]
$$

appearing in (11) is positive semidefinite since it is defined by the product

$$
\Omega_{i}=\left[\begin{array}{c}
X_{i}\left(\Phi_{i}^{(N)}\right)^{\top} \\
\left(\Delta_{i}^{\left(M \times n_{\mathrm{p}}\right)}\right)^{\top}
\end{array}\right]\left[\begin{array}{ll}
\Phi_{i}^{(N)} X_{i} & \Delta_{i}^{\left(M \times n_{\mathrm{p}}\right)}
\end{array}\right] .
$$


$g\left(\alpha, \beta^{+}, \beta^{-}\right)=-\frac{1}{2 \gamma_{1}} \alpha^{\top} \alpha+\alpha^{\top} Y-\frac{1}{2} \sum_{i=1}^{n_{\mathrm{g}}}\left[\begin{array}{c}\alpha \\ \beta^{-}-\beta^{+}\end{array}\right]^{\top}\left[\begin{array}{cc}X_{i}\left(\Phi_{i}^{(N)}\right)^{\top} \Phi_{i}^{(N)} X_{i} & X_{i}\left(\Phi_{i}^{(N)}\right)^{\top} \Delta_{i}^{\left(M \times n_{\mathrm{p}}\right)} \\ \left(\Delta_{i}^{\left(M \times n_{\mathrm{p}}\right)}\right)^{\top} \Phi_{i}^{(N)} X_{i} & \left(\Delta_{i}^{\left(M \times n_{\mathrm{p}}\right)}\right)^{\top} \Delta_{i}^{\left(M \times n_{\mathrm{p}}\right)}\end{array}\right]\left[\begin{array}{c}\alpha \\ \beta^{-}-\beta^{+}\end{array}\right]$

\section{Estimation of the coefficient functions}

Since the feature maps $\phi$ are assumed to be a priori unknown, the parameter vector $\omega_{i}$ can not be explicitly computed via (10a). On the other hand, thanks to the structure of the Lagrange dual function (11), the dual problem can be solved without a prior specification of the feature maps defining the matrices $\Phi_{i}^{(N)}$ and $\Delta_{i}^{\left(M \times n_{\mathrm{p}}\right)}$. Let us first rewrite the matrix $\Omega_{i}$ in the block form

$$
\begin{aligned}
\Omega_{i} & =\left[\begin{array}{cc}
X_{i}\left(\Phi_{i}^{(N)}\right)^{\top} \Phi_{i}^{(N)} X_{i} & X_{i}\left(\Phi_{i}^{(N)}\right)^{\top} \Delta_{i}^{\left(M \times n_{\mathrm{p}}\right)} \\
\left(\Delta_{i}^{\left(M \times n_{\mathrm{p}}\right)}\right)^{\top} \Phi_{i}^{(N)} X_{i} & \left(\Delta_{i}^{\left(M \times n_{\mathrm{p}}\right)}\right)^{\top} \Delta_{i}^{\left(M \times n_{\mathrm{p}}\right)}
\end{array}\right] \\
& =\left[\begin{array}{cc}
\Omega_{i}^{(N, N)} & \Omega_{i}^{\left(N, M \times n_{\mathrm{p}}\right)} \\
\left(\Omega_{i}^{\left(N, M \times n_{\mathrm{p}}\right)}\right)^{\top} & \Omega_{i}^{\left(M \times n_{\mathrm{p}}, M \times n_{\mathrm{p}}\right)}
\end{array}\right]
\end{aligned}
$$

The entries of $\Omega_{i}^{(N, N)}$ are given by

$$
\begin{aligned}
{\left[\Omega_{i}^{(N, N)}\right]_{j, k} } & =x(j) \phi_{i}^{\top}(p(j)) \phi_{i}(p(k)) x(k) \\
& =x(j) K_{i}(p(j), p(k)) x(k),
\end{aligned}
$$

where $K_{i}$ is a positive definite kernel function defining the inner products of $\phi_{i}^{\top}(j) \phi_{i}(k)$. Consequently, $K_{i}$ defines $\Omega_{i}$ and hence characterizes the feature maps $\left\{\phi_{i}\right\}_{i=1}^{n_{\mathrm{g}}}$ in an efficient fashion. This allows to characterize a wide range of nonlinear dependencies as a linear combination of infinitely many functions $\left(n_{\mathrm{H}}=\infty\right)$ defined through the choice of the particular inner product and a relatively low dimensional parameter $\alpha$. This approach is called the kernel trick [11], [12] and in fact it corresponds to function estimator based on the reproducing kernel Hilbert space theory [13].

The matrix $\Omega_{i}^{\left(N, M \times n_{\mathrm{p}}\right)}$ is defined as

$$
\Omega_{i}^{\left(N, M \times n_{\mathrm{p}}\right)}=\left[\begin{array}{llll}
J_{i}\left(\bar{p}_{1}\right) & J_{i}\left(\bar{p}_{2}\right) & \ldots & J_{i}\left(\bar{p}_{M}\right)
\end{array}\right],
$$

where the entries of $J_{i}(\bar{p})$ are given by

$$
\left[J_{i}^{\left(N, n_{\mathrm{p}}\right)}(\bar{p})\right]_{j, k}=x(j) \phi_{i}^{\top}(p(j)) \frac{\partial \phi(\bar{p})}{\partial p_{k}}=x(j) \frac{\partial K_{i}(p(j), \bar{p})}{\partial p_{k}} .
$$

Finally, the matrix $\Omega_{i}^{\left(M \times n_{\mathrm{p}}, M \times n_{\mathrm{p}}\right)}$ is defined as

$$
\begin{aligned}
& \Omega_{i}^{\left(M \times n_{\mathrm{p}}, M \times n_{\mathrm{p}}\right)}= \\
& {\left[\begin{array}{cccc}
H_{i}\left(\bar{p}_{1}, \bar{p}_{1}\right) & H_{i}\left(\bar{p}_{2}, \bar{p}_{1}\right) & \ldots & H_{i}\left(\bar{p}_{M}, \bar{p}_{1}\right) \\
H_{i}\left(\bar{p}_{1}, \bar{p}_{2}\right) & H_{i}\left(\bar{p}_{2}, \bar{p}_{2}\right) & \ldots & H_{i}\left(\bar{p}_{M}, \bar{p}_{2}\right) \\
\vdots & \vdots & \ddots & \vdots \\
\left.H_{i}^{(} \bar{p}_{1}, \bar{p}_{M}\right) & H_{i}\left(\bar{p}_{2}, \bar{p}_{M}\right) & \ldots & H_{i}\left(\bar{p}_{M}, \bar{p}_{M}\right)
\end{array}\right],}
\end{aligned}
$$

where entries of $H_{i}\left(\bar{p}_{1}, \bar{p}_{2}\right)$ are given by

$$
\left[H_{i}^{\left(n_{\mathrm{p}}, n_{\mathrm{p}}\right)}\left(\bar{p}_{1}, \bar{p}_{2}\right)\right]_{j, k}=\frac{\partial \phi^{\top}\left(\bar{p}_{1}\right)}{\partial p_{j}} \frac{\partial \phi\left(\bar{p}_{2}\right)}{\partial p_{k}}=\frac{\partial K_{i}\left(\bar{p}_{1}, \bar{p}_{2}\right)}{\partial p_{j} \partial p_{k}} .
$$

It can be noticed that the Kernel not only characterizes the feature maps, but is also defines their partial derivatives w.r.t. to the scheduling variables.

For example, a typical choice of kernel is the radial basis function (RBF) kernel:

$$
K_{i}(p(j), p(k))=\exp \left(-\frac{\|p(j)-p(k)\|_{\ell_{2}}^{2}}{\sigma_{i}^{2}}\right) .
$$

Using RBFs as the kernel in the simplest case where $n_{\mathrm{p}}=$ 1 , the derivatives in (19) and (21) become:

$$
\begin{aligned}
\frac{\partial K_{i}\left(s_{1}, s_{2}\right)}{\partial s_{2}} & =\frac{2\left(s_{1}-s_{2}\right)}{\sigma_{i}^{2}} K_{i}\left(s_{1}, s_{2}\right), \\
\frac{\partial^{2} K_{i}\left(s_{1}, s_{2}\right)}{\partial s_{1} \partial s_{2}} & =\left[\frac{2}{\sigma_{i}^{2}}-\frac{4\left(s_{1}-s_{2}\right)^{2}}{\sigma_{i}^{4}}\right] K_{i}\left(s_{1}, s_{2}\right) .
\end{aligned}
$$

Note that other choice of kernel functions supply other partial derivatives, while in the RBF case, these partial derivatives corresponds to the first and second moments of a multidimensional Gaussian distribution.

With the use of this kernel trick, the Lagrangian multipliers $\alpha, \beta^{+}, \beta^{-}$can be computed through the dual problem (13) without specifying the feature maps $\phi_{i}$. When we combine the optimality condition (10a) and the definition of the kernel function, we can use these Lagrangian multipliers to estimate the coefficient functions:

$$
\begin{aligned}
a_{i}(\cdot)=\phi_{i}(\cdot)^{\top} \omega_{i}=\sum_{k=1}^{N} \alpha_{k} K_{i}(\cdot, p(k)) x_{i}(k) \\
\quad+\sum_{j=1}^{n_{\mathrm{p}}} \sum_{m=1}^{M} \frac{\partial K_{i}\left(\cdot, \bar{p}_{m}\right)}{\partial p_{j}}\left(\beta_{i, j, m}^{-}-\beta_{i, j, m}^{+}\right) .
\end{aligned}
$$

With these estimated coefficient functions, it becomes possible to find the underlying dependency structure of the data generating process. Because of the regulation term, all coefficient functions without a dependency on a particular dimension of $p$ will be forced to zero along that dimension. By using a threshold value on these relationships, these dependency structures give valuable knowledge that can be further used in identification steps. Furthermore, due to the shrinkage on the variation of the estimated coefficient functions, increasing the $\gamma_{2}$ hyper-parameter will directly result in enforcing a smoother estimate of the dependencies wherever it is possible without significantly increasing the $\ell_{2}$ loss on the prediction. This results in a gradual decrease of the complexity of the estimated functions. 


\section{Simulation EXAMPLE}

The performance of the approach w.r.t. recovering the structural dependence of an LPV system is shown in this section by means of a Monte-Carlo study based simulation example. In this study, we assume that $p(k)$ is scalar (i.e., $\left.n_{\mathrm{p}}=1\right)$.

The considered asymptotically stable LPV data-generating system is described by the difference equation

$$
\begin{aligned}
y(k)= & a_{1}(p(k)) y(k-1)+a_{2}(p(k)) y(k-2) \\
& +a_{5}(p(k)) y(k-5)+a_{8}(p(k)) y(k-8) \\
+ & b_{2}(p(k)) u(k-2)+b_{7}(p(k)) u(k-7)+e(k),
\end{aligned}
$$

where $e(k)$ is a zero-mean white Gaussian noise and $u(k)$ is the input signal. The coefficients functions are described by the (nonlinear) functions:

$$
\begin{aligned}
& a_{1}(p(k))=-0.2, \\
& a_{2}(p(k))=0.2+0.22 \sin \left(\frac{2}{\pi} p(k)\right), \\
& a_{5}(p(k))=0.15, \\
& a_{8}(p(k))= \begin{cases}-0.4 & \text { if } \quad p(k)>0.4 ; \\
-p(k) & \text { if } \quad-0.4 \leq p(k) \leq 0.4 ; \\
0.5 & \text { if } \quad p(k)<-0.4 ;\end{cases} \\
& b_{2}(p(k))=0.5, \\
& b_{7}(p(k))=\exp (-p(k)) .
\end{aligned}
$$

Note that $a_{1}, a_{5}$ and $b_{2}$ do not have a dependency on the scheduling variable $p$.

The system described in (25) is estimated from a data set $\mathcal{D}_{N}=\{(u(k), p(k), y(k))\}_{k=1}^{N}$ with $N=500$ input, output, and scheduling-variable measurements. To gather data, the input and the scheduling trajectories have been chosen to be white-noise sequences, independent of each other, and with a uniform distribution $\mathcal{U}(-1,1)$. In order to provide representative results, a Monte-Carlo simulation of 100 runs is performed. At each run, new realizations of the noise, of the input and of the scheduling variable are considered. The average of the signal-to-noise ratio (SNR) over the MonteCarlo simulation is equal to $23 \mathrm{~dB}$, where the $\mathrm{SNR}$ is defined, at each run, as

$$
10 \log _{10}\left(\frac{\sum_{k=1}^{N} w(k)^{2}}{\sum_{k=1}^{N} e_{0}(k)^{2}}\right)
$$

with $w(k)$ denoting the noise-free system output.

The following LPV model structure is used to estimate the data-generating system:

$$
y(k)=\sum_{i=1}^{n_{\mathrm{a}}} a_{i}(p(k)) y(k-i)+\sum_{j=1}^{n_{\mathrm{b}}} b_{j}(p(k)) u(k-j),
$$

with $n_{\mathrm{a}}=n_{\mathrm{b}}=10$. In the model structure, the dependencies of $a_{i}(p(k))$ and $b_{j}(p(k))$ on the scheduling variable $p$ are not specified.

The coefficient functions $a_{i}(p(k))$ are estimated in two ways: by means of a non-regularized LS-SVM approach, corresponding to the minimization of the objective function (4) for $\gamma_{2}=0$ and by using the regularized LS-SVM (RLSSVM) approach described in this paper. In both approaches, radial basis functions are used as kernels to define the inner product among the feature maps. The values of the parameters $\gamma_{1}$ and $\gamma_{2}$ are chosen via cross-validation based grid search, independently for each method. The interval $\mathbb{P}$ $=\left[\begin{array}{ll}-1 & 1\end{array}\right]$ is gridded into 13 equidistant nodes $\mathcal{P}=\left\{\bar{p}_{m}\right\}_{m=1}^{13}$.

To compare the regularized LS-SVM to the nonregularized method, the average (over the Monte Carlo simulation) of the estimated peak to peak values of the coefficient functions is computed. These values are reported in Table I. Here we see that the RLS-SVM method is able estimate the coefficient functions with a reduced bias and variance in comparison with the LS-SVM method. The small mean values of the non-dependent coefficient functions show that it is possible to extract information about the dependency structure of the underlying process. This can be further exploited via thresholding based re-estimation.

In order to visualize the difference between the LS-SVM and RLS-SVM methods, the difference between the true coefficient functions and the coefficient functions estimated with the two methods is plotted in Fig. 1, together with the standard deviation boundaries. The estimated values are the mean values of the 100 Monte-Carlo runs. The error reduction by using the RLS-SVM is clearly visible and it can also be seen that the recovered coefficient functions $a_{1}, a_{5}$ and $b_{2}$ do not have any dependency on $p$. This knowledge could be further used in order to better parameterize the model and hence reduce the variance.

\section{CONCLUSIONS}

In this paper, we have addressed the problem of identifying LPV-ARX models in the LS-SVM framework, where the underlying dependence of the coefficient functions on the scheduling variable is not a priori known. By penalizing the sensitivity of the coefficient functions w.r.t. the scheduling variables, the method provides a way to estimate the dependency structure of the plant without exploiting any a priori information on the underlying behavior of the true LPV system. The reported simulation has shown the effectiveness of the proposed approach in providing a lowervariance estimate (w.r.t. to standard LS-SVM methods) of the coefficient functions describing the LPV model.

\section{REFERENCES}

[1] J. S. Shamma and M. Athans, "Analysis of gain scheduled control for nonlinear plants," IEEE Trans. on Automatic Control, vol. 35, no. 8, pp. 898-907, 1990.

[2] R. Tóth, Modeling and Identification of Linear Parameter-Varying Systems, ser. Lecture Notes in Control and Information Sciences, Vol. 403. Heidelberg: Springer, 2010.

[3] J. Suykens, T. Van Gestel, J. De Brabanter, B. De Moor, and J. Vandewalle, Least Squares Support Vector Machines. World Scientific, 2002.

[4] R. Tóth, V. Laurain, W. Zheng, and K. Poolla, "Model structure learning: A support vector machine approach for LPV linear-regression models," in Proc. of the 50th IEEE Conf. on Decision and Control, Orlando, Florida, USA, Dec. 2011, pp. 3192-3197. 
TABLE I

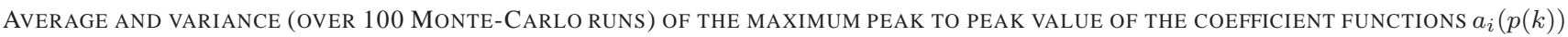
AND $b_{j}(p(k))$ ESTIMATED BY MEANS OF THE LS-SVM AND RLS-SVM APPROACH.

\begin{tabular}{|c|c|c|c|c|c|c|c|c|c|c|}
\hline True value & $\begin{array}{c}\overline{a_{1}} \\
0\end{array}$ & $\begin{array}{c}\overline{a_{2}} \\
0.22\end{array}$ & $\begin{array}{c}\overline{a_{3}} \\
0\end{array}$ & $\begin{array}{c}\overline{a_{4}} \\
0\end{array}$ & $\begin{array}{c}\overline{a_{5}} \\
0\end{array}$ & $\begin{array}{c}\overline{a_{6}} \\
0\end{array}$ & $\begin{array}{c}\overline{a_{7}} \\
0\end{array}$ & $\begin{array}{l}\overline{a_{8}} \\
0.9\end{array}$ & $\begin{array}{c}\overline{a_{9}} \\
0\end{array}$ & $\begin{array}{c}a_{10}^{-} \\
0\end{array}$ \\
\hline Mean (RLS-SVM) & $2.1 \cdot 10^{-5}$ & $2.0 \cdot 10^{-1}$ & $2.2 \cdot 10^{-3}$ & $3.8 \cdot 10^{-8}$ & $8.6 \cdot 10^{-4}$ & $2.2 \cdot 10^{-8}$ & $3.1 \cdot 10^{-4}$ & $8.70 \cdot 10^{-1}$ & $3.7 \cdot 10^{-4}$ & $2.7 \cdot 10^{-8}$ \\
\hline Mean (LS-SVM) & $8.8 \cdot 10^{-2}$ & $1.7 \cdot 10^{-1}$ & $9.4 \cdot 10^{-2}$ & $4.8 \cdot 10^{-2}$ & $1.4 \cdot 10^{-1}$ & $6.6 \cdot 10^{-2}$ & $7.3 \cdot 10^{-2}$ & $5.4 \cdot 10^{-1}$ & $5.8 \cdot 10^{-2}$ & $4.5 \cdot 10^{-2}$ \\
\hline Var (RLS-SVM) & $5.8 \cdot 10^{-10}$ & $8.4 \cdot 10^{-4}$ & $4.2 \cdot 10^{-5}$ & $2.7 \cdot 10^{-15}$ & $1.2 \cdot 10^{-5}$ & $2.9 \cdot 10^{-16}$ & $2.0 \cdot 10^{-6}$ & $8.4 \cdot 10^{-4}$ & $7.1 \cdot 10^{-6}$ & $7.1 \cdot 10^{-16}$ \\
\hline Var (LS-SVM) & $1.1 \cdot 10^{-3}$ & $8.7 \cdot 10^{-4}$ & $1.3 \cdot 10^{-3}$ & $8.5 \cdot 10^{-4}$ & $1.5 \cdot 10^{-3}$ & $8.9 \cdot 10^{-4}$ & $8.6 \cdot 10^{-4}$ & $2.4 \cdot 10^{-3}$ & $9.3 \cdot 10^{-4}$ & $5.8 \cdot 10^{-4}$ \\
\hline & $\overline{b_{1}}$ & $\overline{b_{2}}$ & $\overline{b_{3}}$ & $\overline{b_{4}}$ & $\overline{b_{5}}$ & $\overline{b_{6}}$ & $\overline{b_{7}}$ & $\overline{b_{8}}$ & $\overline{b_{9}}$ & $\overline{b_{10}^{-}}$ \\
\hline True value & 0 & 0 & 0 & 0 & 0 & 0 & 2.35 & 0 & 0 & 0 \\
\hline Mean (RLS-SVM) & $2.2 \cdot 10^{-8}$ & $3.8 \cdot 10^{-5}$ & $2.4 \cdot 10^{-8}$ & $3.1 \cdot 10^{-8}$ & $2.6 \cdot 10^{-8}$ & $2.3 \cdot 10^{-8}$ & $1.8 \cdot 10^{0}$ & $4.1 \cdot 10^{-8}$ & $6.6 \cdot 10^{-8}$ & $7.2 \cdot 10^{-8}$ \\
\hline Mean (LS-SVM) & $4.6 \cdot 10^{-2}$ & $2.1 \cdot 10^{-1}$ & $4.3 \cdot 10^{-2}$ & $4.0 \cdot 10^{-2}$ & $4.5 \cdot 10^{-2}$ & $4.4 \cdot 10^{-2}$ & $8.5 \cdot 10^{-1}$ & $4.1 \cdot 10^{-2}$ & $5.8 \cdot 10^{-2}$ & $4.6 \cdot 10^{-2}$ \\
\hline Var (RLS-SVM) & $4.5 \cdot 10^{-16}$ & $3.3 \cdot 10^{-10}$ & $6.6 \cdot 10^{-16}$ & $7.1 \cdot 10^{-16}$ & $6.0 \cdot 10^{-16}$ & $3.4 \cdot 10^{-16}$ & $5.3 \cdot 10^{-3}$ & $2.2 \cdot 10^{-15}$ & $3.0 \cdot 10^{-15}$ & $4.1 \cdot 10^{-15}$ \\
\hline $\operatorname{Var}(\mathrm{LS}-\mathrm{SVM})$ & $8.7 \cdot 10^{-4}$ & $6.9 \cdot 10^{-4}$ & $7.6 \cdot 10^{-4}$ & $5.8 \cdot 10^{-4}$ & $9.4 \cdot 10^{-4}$ & $6.4 \cdot 10^{-4}$ & $3.8 \cdot 10^{-3}$ & $4.3 \cdot 10^{-4}$ & $9.7 \cdot 10^{-4}$ & $7.5 \cdot 10^{-4}$ \\
\hline
\end{tabular}

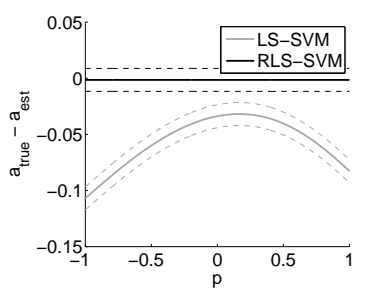

(a) $a_{1}$

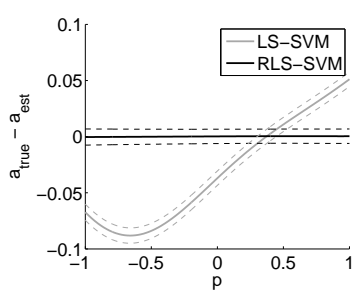

(c) $a_{5}$

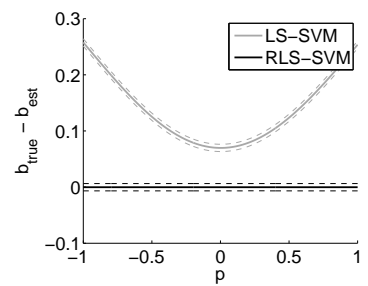

(e) $b_{2}$

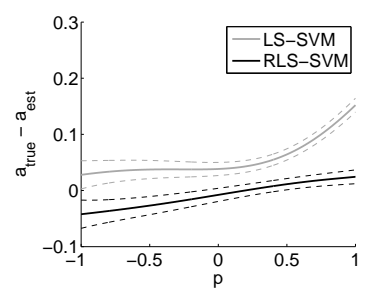

(b) $a_{2}$

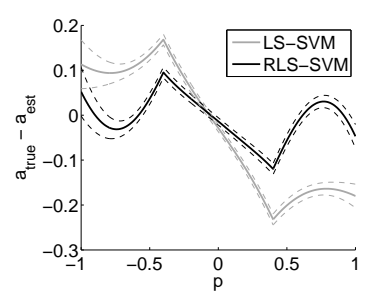

(d) $a_{8}$

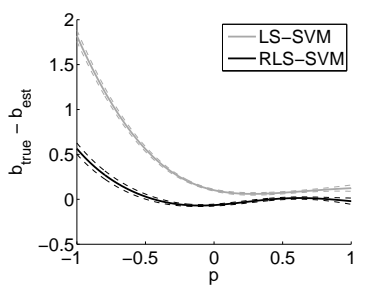

(f) $b_{7}$
Fig. 1. Difference between the true and the estimated coefficient functions together with the standard deviation boundaries.

[5] V. Laurain, R. Tóth, W. Zheng, and M. Gilson, "Nonparametric identification of LPV models under general noise conditions. an LSSVM based approach," in Proc. of the the 16th IFAC Symposium on System Identification, Brussels, Belgium, July 2012, pp. 1761-1766.

[6] D. Piga and R. Tóth, "LPV model order selection in an LS-SVM setting," in Proc. of the 52nd IEEE Conference on Decision and Control, Florence, Italy, Dec. 2013, pp. 4128-4133.

[7] L. Giarré, D. Bauso, P. Falugi, and B. Bamieh, "LPV model identification for gain scheduling control: An application to rotating stall and surge control problem," Control Engineering Practice, vol. 14, no. 4, pp. 351-361, 2006.

[8] J. W. van Wingerden and M. Verhaegen, "Subspace identification of bilinear and LPV systems for open- and closed-loop data," Automatica, vol. 45, no. 2, pp. 372-381, 2009.

[9] T. Falck, K. Pelckmans, J. Suykens, and B. De Moor, "Identification of wiener-hammerstein systems using LS-SVMs," in 15th IFAC symposium on System Identification, Saint Malo, France, July 2009.

[10] I. Goethals, K. Pelckmans, J. Suykens, and B. De Moor, "Identification of MIMO Hammerstein models using least squares support vector machines," Automatica, vol. 41, no. 7, pp. 1263-1272, 2005.

[11] B. Schölkopf and A. Smola, Learning with kernels. Cambridge MA: MIT Press, 2002

[12] V. Vapnik, Statistical Learning Theory. Wiley-Interscience, 1998.

[13] F. Cucker and S. Smale, "On the mathematical foundations of learning," Bulletin of the American Mathematical Society, vol. 39, no. 1, pp. 1-49, 2001. 